\title{
Circulating exosomal IncRNAs in patients with chronic coronary syndromes
}

Meili Zheng ${ }^{1,2}$, Ruijuan $\mathrm{Han}^{3}$, Wen Yuan ${ }^{4}$, Hongjie Chi ${ }^{1,2}$, Yeping Zhang ${ }^{1,2}$, Kai Sun ${ }^{5}$, Jiuchang Zhong ${ }^{1,2,4}$, Xiaoyan Liu ${ }^{1,2,4}$, Xinchun Yang ${ }^{1,2}$

\author{
${ }^{1}$ Heart Center, Beijing Chao-Yang Hospital, Capital Medical University, Beijing, China \\ ${ }^{2}$ Beijing Key Laboratory of Hypertension Research, Beijing Chao-Yang Hospital, \\ Capital Medical University, Beijing, China \\ ${ }^{3}$ Department of Radiology, State Key Laboratory of Cardiovascular Disease, Fu Wai \\ Hospital, National Center for Cardiovascular Diseases, Chinese Academy of Medical \\ Sciences and Peking Union Medical College, Beijing, China \\ ${ }^{4}$ Medical Research Center, Beijing Chao-Yang Hospital, Capital Medical University, \\ Beijing, China \\ ${ }^{5}$ Department of Radiology, Fuwai Hospital Chinese Academy of Medical Sciences, \\ Shenzhen, China
}

Submitted: 3 July 2020; Accepted: 1 October 2020

Online publication: 8 January 2021

Arch Med Sci 2023; 19 (1): 46-56

DOI: https://doi.org/10.5114/aoms/128014

Copyright $\odot 2020$ Termedia \& Banach

\section{Abstract}

Introduction: The concept of chronic coronary syndrome (CCS) was first presented at the European Society of Cardiology Meeting in 2019. However, the roles of exosomal IncRNAs in CCS remain largely unclear.

Material and methods: A case-control study was performed with a total of 218 participants (137 males and 81 females), including 15 CCS patients and 15 controls for sequencing profiles, 20 CCS patients and 20 controls for the first validation, and 100 CCS patients and 48 controls for the second validation. Exosomes were isolated from the plasma of CCS patients and controls, and exosomal IncRNAs were identified by sequencing profiles and verified twice by qRT-PCR analysis. Receiver operating characteristic (ROC) curve analysis was performed to evaluate the diagnostic value of exosomal IncRNAs for CCS patients. Results: A total of 152 significantly differentially expressed IncRNAs with over two-fold changes were detected in plasma exosomes of CCS patients, including 90 upregulated and 62 downregulated IncRNAs. Importantly, 6 upregulated IncRNAs with the top fold changes were selected for validations. Exosomal IncRNAs ENST00000424615.2 and ENST00000560769.1 were significantly elevated in CCS patients in both validations compared with controls. The areas under the ROC of IncRNAs ENST00000424615.2 and ENST00000560769.1 were 0.654 and 0.722 , respectively. Additionally, exosomal IncRNA ENST00000560769.1 was significantly higher in the CCS patients with more diseased vessels $(p=0.028)$. Conclusions: Exosomal IncRNA ENST00000424615.2 and ENST00000560769.1 were identified as novel diagnosis biomarkers for patients with CCS. Moreover, exosomal IncRNA ENST00000560769.1 was significantly higher in the CCS patients with more diseased vessels, and might be associated with a poor prognosis.

Key words: exosomal IncRNAs, chronic coronary syndrome, coronary artery disease, biomarkers.

\section{Introduction}

Coronary artery disease (CAD) is a pathological process characterized by obstructive or non-obstructive atherosclerotic plaque accumulation in

\author{
Corresponding authors: \\ Prof. Xinchun Yang, \\ Dr. Xiaoyan Liu, \\ and Prof. Jiuchang Zhong \\ Beijing Chao-Yang Hospital \\ Capital Medical University \\ 8\# Gong-Ti South Road \\ Beijing, China \\ Phone: 86-10-85231066 \\ Fax: 86-10-65951064 \\ E-mails: \\ yangxc99@gmail.com (X. Yang); \\ lxy-213@163.com (X. Liu); \\ jczhong@sina.com (J. Zhong)
}


the epicardial arteries. The disease can have long, stable periods but can also become unstable at any time, typically due to an acute atherothrombotic event caused by plaque rupture or erosion, which can present clinically as either chronic coronary syndrome (CCS) or acute coronary syndrome (ACS) [1]. Acute coronary syndrome is a medical emergency requiring immediate intervention; CCS also needs to be taken seriously since CAD is often progressive even in apparently clinically silent periods.

In the development and progression of CAD, emerging evidence suggests that exosomes have played crucial roles [2]. Exosomes are small membrane microvesicles (30-120 nm), produced by almost all cell types, that deliver their cargoes (proteins, DNA, and non-coding RNAs) to be involved in intercellular communication [3]. Recent studies have shown that exosomes might play important roles in cardiovascular diseases, and exosomal cargoes emerged as biomarkers or for use in therapeutic approaches [4-6]. Exosomal miRNAs have been reported to be potential biomarkers in ACS [7]; and exosomal IncRNAs may be involved in atherosclerosis progression [8-10], which is the key prerequisite for CCS. Several studies have investigated the role of IncRNAs serving as biomarkers to diagnose CAD [11-14], however, scarce research has been performed on the role of exosomal IncRNAs in CCS patients.

In the present study, we compared the expression of circulating exosomal IncRNAs in CCS patients and control subjects, and investigated the relationship between exosomal IncRNAs and the clinical parameters in CCS patients. Our findings indicate a potential role of circulating exosomal IncRNAs in CCS.

\section{Material and methods}

\section{Study subjects}

This is a case-control study. All CCS cases and control subjects were consecutively enrolled from Beijing Chao-Yang Hospital (Capital Medical University, Beijing, China) between January and December 2017. All the participants in this investigation had been informed and had given written consent. The study was conducted in accordance with the Declaration of Helsinki, and the research protocol was approved by the Ethics Committee of Beijing Chao-Yang Hospital (Capital Medical University, Beijing, China).

Chronic coronary syndrome was defined according to the European Society of Cardiology (ESC) Clinical Practice Guidelines in 2019 [1]. Six subcategories of CCS were included: suspected CAD with stable anginal symptoms and or dyspnea (anginal equivalent); new onset heart failure or left ventricular dysfunction and suspected coronary artery disease; asymptomatic and symptomatic patients with stabilized symptoms occurring less than 1 year after an acute coronary syndrome or patients with recent revascularization; asymptomatic and symptomatic patient more than 1 year after initial diagnosis or revascularization; patients with angina and suspected microvascular disease or vasospastic angina; and asymptomatic persons in whom CAD was detected at screening. All CCS patients included in the present study were those with coronary vessel stenosis $\geq 50 \%$ on coronarography. The control subjects were non-coronary chest pain patients (NCCP), with chest pain, normal cardiac biomarkers and most importantly, no coronary stenosis as confirmed by coronarography.

The following exclusion criteria were applied: malignant diseases, severe organ failure, thyroid dysfunction, autoimmune diseases, and various acute or chronic infectious diseases, and pregnancy.

A total of 218 participants (137 males and $81 \mathrm{fe-}$ males) were included in the present study, and all the study subjects were divided into three groups: a sequencing group (subjects' blood samples for exosomal RNA sequencing), including 15 CCS and 15 controls (for sequencing profiles, the blood samples of every five subjects were pooled into one collective sample, and thus "3 CCS" and "3 control" samples were profiled); the first validation group (samples for exosomal RNA validation were taken for the first time), including 20 CCS and 20 controls; the second validation group (samples for the second validation were collected), including 100 CCS and 48 controls.

\section{Exosome experiments}

Exosomal isolation and identification were performed. Exosomal isolation was done following the instructions of the commercial kit used (Qiagen Inc., Dusseldorf, Germany). Isolated exosomes were identified by transmission electron microscopy (TEM), nanoparticle tracking analysis (NTA), and Western blotting (WB) of the exosomal marker protein CD63 (Abcam, Cambridge, UK) and HSP70 (Abcam, Cambridge, UK) as described previously [15-17].

\section{Exosomal RNA experiments}

Exosomal RNA experiments included exosomal RNA extraction, sequencing, and validation by quantitative RT-PCR (qRT-PCR). Exosomal RNA extraction was carried out according to the protocol of the commercial kit utilized (Qiagen Inc., Dusseldorf, Germany). RNA sequencing was done as described in the methods section of our previous study [18]. The relative expression levels of IncRNA were quantified using ViiA 7 Real-Time PCR System (Applied Biosystems, Foster City, CA, USA) based on standard methods. The forward and reverse primers used are listed in Table I (the IncRNA IDs were searched in Ensembl Human GRCh37.p13). 
Table I. Forward and reverse primers

\begin{tabular}{|lcc|}
\hline $\begin{array}{l}\text { IncRNA ID } \\
\text { (Ensembl Human GRCh37.p13) }\end{array}$ & Forward primer (5'-3') & Reverse primer $\mathbf{( 5}^{\prime} \mathbf{-} \mathbf{3}^{\prime} \mathbf{)}$ \\
\hline ENST00000424615.2 & CTCTGAAAGAACTCTCACATGGACC & GTCCTCTCCTTTGATGGACTCC \\
\hline NR_037611.1 & CCAGTCCTACCCTGGAACCTT & CCAGTAGAATCCCAACAGGCA \\
\hline ENST00000528983.1 & GTGAGCAAGGTGCCGTTTT & CCAGCCTCCACACTGCATTAA \\
\hline ENST00000560453.1 & CCCAGTTGGCTGACGAAGA & GCAGGTGTAGGTGGTAGTTGAAG \\
\hline ENST00000593793.1 & AAAGCGGAGTCCATCAAAGG & CGGTCTGAACGGTGTGTTATCT \\
\hline ENST00000560769.1 & AAGGACTTGCTGGGTGTGCT & CCAAAGACGGGTTTCATTAGGT \\
\hline Hsa-18S & CATTCGAACGTCTGCCCTAT & GCCTTCCTTGGATGTGGTAG \\
\hline
\end{tabular}

Laboratory measurements, echocardiography, and coronary artery angiography

Peripheral venous blood samples were collected after overnight fasting in the first $24 \mathrm{~h}$ after admission before coronary artery angiography (CAG) to measure a range of parameters, including lipids, glucose, and creatinine. Blood samples with a volume of $8 \mathrm{ml}$ (collected into EDTA-containing tubes) were retained to obtain plasma for exosome isolation, and were then stored at $-80^{\circ} \mathrm{C}$ until further use. Echocardiography was performed to measure routine parameters, such as the left ventricular end diastolic diameter (LVEDD), left ventricular ejection fraction (LVEF), etc. CAG was performed within $72 \mathrm{~h}$ after admission in both CCS patients and control subjects.

\section{Statistical analysis}

Statistical analysis was performed using SPSS 24.0 software (IBM, Armonk, NY, USA). All statistical analyses were two-tailed, and $p$-values $<0.05$ were considered statistically significant. Continuous variables with normal distribution were expressed as mean \pm standard deviation (SD), and compared by Student's $t$-test, whereas those with non-normal distribution were expressed as quartiles and compared by the Mann-Whitney U-test. Categorical variables were expressed as percentages and numbers, and compared using the $\chi^{2}$ test. Spearman correlation was used to calculate the correlation between exosomal IncRNAs and clinical parameters. Receiver operating characteristic curves and areas under the curve (AUCs) were computed to estimate the effectiveness of exosomal IncRNAs for prediction.

\section{Results}

Sequence profiling of circulating exosomal IncRNA

The present study included 15 CCS patients and 15 control subjects, whose clinical characteristics are summarized in Table II. No significant differences were detected in the subject characteristics between the two groups; they were well balanced with regard to the main clinical and laboratory characteristics.

Before sequence profiling of circulating exosomal IncRNA, exosomes from the plasma were identified by TEM analyses and NTA and WB experiments (Supplementary Figure S1). TEM showed that the exosomes had a spherical shape with a bilayer membrane vesicle structure. The diameter of each of the exosome established by NTA was approximately $100 \mathrm{~nm}$. The results of WB experiments suggested that specific proteins, CD63 and HSP70, were positively expressed in the exosomes in both groups.

By sequence profiling, we detected a total number of 152 significantly differentially expressed IncRNAs ( $p<0.05$; fold change $>2$ ) in the plasma exosomes of the two groups, including 90 upregulated and 62 downregulated. Of the 90 upregulated IncRNA identified, we selected six highly expressed IncRNAs with the top fold change (IncRNA ENST00000424-615.2, IncRNA NR_037611.1, IncRNA ENST0000052-8983.1, IncRNA ENST00000560453.1, IncRNA ENSTO0000593793.1, and IncRNAENST0000056-0769.1) to perform the subsequent first validation (Figure 1).

\section{First validation of circulating exosomal IncRNA}

The first validation was performed in 20 CCS patients and 20 control subjects, whose clinical characteristics are presented in Table III. The results of the first validation of the six selected circulating exosomal IncRNAs (ENST00000424615.2, NR_037611.1, ENST00000528983.1, ENST00000560453.1, ENST00000593793.1, and ENST00000560769.1) showed that IncRNA ENST00000424615.2 ( $p<0.001$, fold change $=2.49$ ) and IncRNA ENST00000560769.1 $(p<0.001$, fold change $=$ 3.17) were significantly upregulated in AMI patients in the first validation. On the other hand, IncRNANR_037611.1 $(p=0.990$, fold change $=1.67)$, IncRNA ENST00000528983.1 $(p=0.050$, fold change $=1.99$ ), IncRNA ENST00000560453.1 $(p=0.560$, fold change $=4.50)$, and IncRNA 
Table II. Clinical characteristics of sequencing samples

\begin{tabular}{|c|c|c|c|}
\hline Parameter & Control, $n=15$ & CCS, $n=15$ & $P$-value \\
\hline Age [years] & $55.4 \pm 9.9$ & $60.9 \pm 8.0$ & 0.105 \\
\hline Male, $n(\%)$ & $8(53.3)$ & $10(66.6)$ & 0.711 \\
\hline Hypertension, $n$ (\%) & $7(46.7)$ & $11(73.3)$ & 0.265 \\
\hline Diabetes, $n(\%)$ & $1(6.7)$ & $0(0.0)$ & 0.996 \\
\hline Current smoker, $n(\%)$ & $5(33.3)$ & $4(26.7)$ & 0.997 \\
\hline Current drinker, $n(\%)$ & $4(26.7)$ & $2(13.3)$ & 0.645 \\
\hline Family history, $n$ (\%) & $4(26.7)$ & $3(13.3)$ & 0.645 \\
\hline Heart rate [beats/min] & $68.0(60.0-77.0)$ & $66.0(57.0-78.0)$ & 0.274 \\
\hline Systolic blood pressure [mm Hg] & $123.1 \pm 34.7$ & $138.9 \pm 17.6$ & 0.127 \\
\hline Diastolic blood pressure [mm Hg] & $74.7 \pm 8.1$ & $82.0 \pm 10.8$ & 0.072 \\
\hline Body mass index $\left[\mathrm{kg} / \mathrm{m}^{2}\right]$ & $26.3 \pm 4.9$ & $24.7 \pm 2.4$ & 0.266 \\
\hline Total cholesterol [mmol/l] & $4.10(3.63-5.22)$ & $4.48(3.50-5.25)$ & 0.838 \\
\hline $\mathrm{HDL}-\mathrm{C}[\mathrm{mmol} / \mathrm{l}]$ & $1.10(1.00-1.59)$ & $1.10(0.90-1.54)$ & 0.870 \\
\hline LDL-C [mmol/l] & $2.70(1.95-3.00)$ & $2.30(2.10-3.20)$ & 0.935 \\
\hline Triglycerides [mmol/l] & $1.12(0.89-1.70)$ & $1.43(1.25-2.02)$ & 0.074 \\
\hline Fast glucose $[\mathrm{mmol} / \mathrm{l}]$ & $4.84(4.39-4.98)$ & $5.04(4.63-5.33)$ & 0.174 \\
\hline
\end{tabular}

$A M I$ - acute myocardial infarction, HDL-C - high-density lipoprotein cholesterol, LDL-C - low-density lipoprotein cholesterol.

Table III. Clinical characteristics of subjects in the first and second validations

\begin{tabular}{|c|c|c|c|c|c|c|}
\hline \multirow[t]{2}{*}{ Parameter } & \multicolumn{3}{|c|}{ First validation } & \multicolumn{3}{|c|}{ Second validation } \\
\hline & $\begin{array}{l}\text { Control } \\
(n=20)\end{array}$ & $\begin{array}{c}\text { CCS } \\
(n=20)\end{array}$ & $P$-value & $\begin{array}{l}\text { Control } \\
(n=48)\end{array}$ & $\begin{array}{c}\text { CCS } \\
(n=100)\end{array}$ & $P$-value \\
\hline Age [years] & $55.9 \pm 11.1$ & $61.1 \pm 6.2$ & 0.080 & $56.6 \pm 10.8$ & $60.2 \pm 8.2$ & 0.044 \\
\hline Male, $n(\%)$ & $9(45.0)$ & $10(50.0)$ & 0.752 & $27(56.3)$ & $73(73.0)$ & 0.042 \\
\hline Current smoker, $n(\%)$ & $8(40.0)$ & $6(30.0)$ & 0.765 & $4(8.3)$ & $49(49.0)$ & $<0.001$ \\
\hline Current drinker, $n$ (\%) & $3(15.0)$ & $3(15.0)$ & 1.000 & $11(22.9)$ & $30(30.0)$ & 0.181 \\
\hline Hospital time [days] & $3.5(3.0-6.0)$ & $4.0(2.0-5.0)$ & 0.327 & $4.0(3.0-6.0)$ & $4.0(3.0-6.0)$ & 0.899 \\
\hline LVEDD [mm] & $\begin{array}{c}45.0 \\
(44.0-49.0)\end{array}$ & $\begin{array}{c}45.0 \\
(42.5-49.5)\end{array}$ & 0.812 & $\begin{array}{c}46.0 \\
(43.0-50.0)\end{array}$ & $\begin{array}{c}48.0 \\
(45.0-50.0)\end{array}$ & 0.046 \\
\hline LVEF (\%) & $\begin{array}{c}71.0 \\
(66.5-72.5)\end{array}$ & $\begin{array}{c}69.0 \\
(68.0-72.0)\end{array}$ & 0.786 & $\begin{array}{c}67.0 \\
(66.0-73.0)\end{array}$ & $\begin{array}{c}68.0 \\
(65.0-72.3)\end{array}$ & 0.850 \\
\hline \multicolumn{7}{|l|}{ Physical data: } \\
\hline Heart rate [beats/min] & $\begin{array}{c}72.0 \\
(65.0-73.0)\end{array}$ & $\begin{array}{c}68.5 \\
(63.3-77.0)\end{array}$ & 0.529 & $\begin{array}{c}71.0 \\
(63.8-79.8)\end{array}$ & $\begin{array}{c}70.0 \\
(62.3-78.0)\end{array}$ & 0.411 \\
\hline $\begin{array}{l}\text { Systolic blood pressure } \\
{[\mathrm{mm} \mathrm{Hg}]}\end{array}$ & $\begin{array}{c}129.5 \\
(119.5-136.0) \\
\end{array}$ & $\begin{array}{c}131.0 \\
(111.3-144.3) \\
\end{array}$ & 0.947 & $\begin{array}{c}128.5 \\
(120.3-140.0) \\
\end{array}$ & $\begin{array}{c}133.0 \\
(120.0-144.5) \\
\end{array}$ & 0.338 \\
\hline $\begin{array}{l}\text { Diastolic blood pressure } \\
{[\mathrm{mm} \mathrm{Hg}]}\end{array}$ & $\begin{array}{c}72.5 \\
(66.3-81.3) \\
\end{array}$ & $\begin{array}{c}74.5 \\
(63.5-80.8) \\
\end{array}$ & 0.738 & $\begin{array}{c}77.5 \\
(70.0-84.8) \\
\end{array}$ & $\begin{array}{c}78.5 \\
(69.0-84.0) \\
\end{array}$ & 0.878 \\
\hline $\begin{array}{l}\text { Body mass index } \\
{\left[\mathrm{kg} / \mathrm{m}^{2}\right]}\end{array}$ & $24.7 \pm 4.2$ & $24.8 \pm 3.9$ & 0.966 & $26.1 \pm 3.5$ & $26.4 \pm 3.4$ & 0.691 \\
\hline \multicolumn{7}{|l|}{ Historical data: } \\
\hline Hypertension, $n$ (\%) & $7(35.0)$ & $12(60.0)$ & 0.113 & $19(39.6)$ & $71(71.0)$ & $<0.001$ \\
\hline Diabetes, $n(\%)$ & $0(0.0)$ & $0(0.0)$ & 1.000 & $5(10.4)$ & $11(11.0)$ & 0.915 \\
\hline Family history, $n(\%)$ & $3(15.0)$ & $6(30.0)$ & 0.449 & $13(27.1)$ & $26(26.0)$ & 0.889 \\
\hline
\end{tabular}


Table III. Cont.

\begin{tabular}{|c|c|c|c|c|c|c|}
\hline \multirow[t]{2}{*}{ Parameter } & \multicolumn{3}{|c|}{ First validation } & \multicolumn{3}{|c|}{ Second validation } \\
\hline & $\begin{array}{l}\text { Control } \\
(n=20)\end{array}$ & $\begin{array}{c}\text { CCS } \\
(n=20)\end{array}$ & $P$-value & $\begin{array}{l}\text { Control } \\
(n=48)\end{array}$ & $\begin{array}{c}\text { CCS } \\
(n=100)\end{array}$ & $P$-value \\
\hline C-reactive protein [mg/l] & $\begin{array}{c}1.51 \\
(0.82-2.24)\end{array}$ & $\begin{array}{c}1.65 \\
(0.68-4.24)\end{array}$ & 0.084 & $\begin{array}{c}1.10 \\
(0.59-2.57)\end{array}$ & $\begin{array}{c}1.29 \\
(0.42-2.43)\end{array}$ & 0.966 \\
\hline $\mathrm{ESR}[\mathrm{mm} / \mathrm{h}]$ & $\begin{array}{c}3.00 \\
(2.00-6.00)\end{array}$ & $\begin{array}{c}6.00 \\
(2.00-10.00)\end{array}$ & 0.100 & $\begin{array}{c}5.00 \\
(2.00-10.50)\end{array}$ & $\begin{array}{c}4.00 \\
(2.00-7.25)\end{array}$ & 0.197 \\
\hline Leukocytes $\left[\times 10^{9} / 1\right]$ & $\begin{array}{c}5.95 \\
(5.46-7.17)\end{array}$ & $\begin{array}{c}7.26 \\
(6.38-7.86)\end{array}$ & 0.035 & $\begin{array}{c}5.90 \\
(5.33-7.25)\end{array}$ & $\begin{array}{c}6.89 \\
(5.58-8.25) \\
\end{array}$ & 0.011 \\
\hline Neutrophils $\left[\times 10^{9} / 1\right]$ & $\begin{array}{c}3.40 \\
(2.89-4.25)\end{array}$ & $\begin{array}{c}4.52 \\
(3.72-5.03)\end{array}$ & 0.033 & $\begin{array}{c}3.73 \\
(3.12-4.52)\end{array}$ & $\begin{array}{c}4.01 \\
(3.21-5.41)\end{array}$ & 0.086 \\
\hline Lymphocytes $\left[\times 10^{9} / I\right]$ & $\begin{array}{c}2.00 \\
(1.65-2.30)\end{array}$ & $\begin{array}{c}1.93 \\
(1.57-2.56)\end{array}$ & 0.799 & $\begin{array}{c}1.67 \\
(1.47-2.07)\end{array}$ & $\begin{array}{c}1.99 \\
(1.56-2.35)\end{array}$ & 0.039 \\
\hline Hemoglobin $[\mathrm{g} / \mathrm{l}]$ & $\begin{array}{c}124.0 \\
(117.5-137.0)\end{array}$ & $\begin{array}{c}129.0 \\
(123.0-138.3)\end{array}$ & 0.547 & $\begin{array}{c}134.5 \\
(124.3-147.8)\end{array}$ & $\begin{array}{c}139.0 \\
(129.0-146.0)\end{array}$ & 0.233 \\
\hline Platelets $\left[\times 10^{9} / 1\right]$ & $\begin{array}{c}246.0 \\
(209.5-266.5)\end{array}$ & $\begin{array}{c}210.5 \\
(188.0-273.8)\end{array}$ & 0.231 & $\begin{array}{c}213.0 \\
(185.5-259.0)\end{array}$ & $\begin{array}{c}216.0 \\
(181.0-247.0)\end{array}$ & 0.874 \\
\hline AST [U/I] & $\begin{array}{c}18.0 \\
(16.0-22.8)\end{array}$ & $\begin{array}{c}19.0 \\
(17.0-21.8)\end{array}$ & 0.620 & $\begin{array}{c}19.0 \\
(17.0-22.5)\end{array}$ & $\begin{array}{c}19.0 \\
(17.0-25.5)\end{array}$ & 0.182 \\
\hline $\mathrm{ALT}[\mathrm{U} / \mathrm{l}]$ & $\begin{array}{c}15.5 \\
(12.0-23.3) \\
\end{array}$ & $\begin{array}{c}20.0 \\
(13.0-24.8) \\
\end{array}$ & 0.461 & $\begin{array}{c}16.5 \\
(12.0-22.0) \\
\end{array}$ & $\begin{array}{c}20.0 \\
(16.0-29.5) \\
\end{array}$ & 0.003 \\
\hline $\begin{array}{l}\text { Total cholesterol } \\
{[\mathrm{mmol} / \mathrm{l}]}\end{array}$ & $\begin{array}{c}4.28 \\
(3.53-4.42) \\
\end{array}$ & $\begin{array}{c}4.09 \\
(3.48-5.10) \\
\end{array}$ & 0.698 & $\begin{array}{c}4.22 \\
(3.58-5.18) \\
\end{array}$ & $\begin{array}{c}3.87 \\
(3.12-4.56) \\
\end{array}$ & 0.072 \\
\hline $\mathrm{HDL}-\mathrm{C}[\mathrm{mmol} / \mathrm{l}]$ & $\begin{array}{c}1.15 \\
(0.93-1.58) \\
\end{array}$ & $\begin{array}{c}1.10 \\
(0.90-1.38)\end{array}$ & 0.620 & $\begin{array}{c}1.10 \\
(0.95-1.30) \\
\end{array}$ & $\begin{array}{c}1.00 \\
(0.80-1.20) \\
\end{array}$ & 0.004 \\
\hline LDL-C [mmol/l] & $\begin{array}{c}2.25 \\
(1.90-2.73) \\
\end{array}$ & $\begin{array}{c}2.50 \\
(1.83-3.25) \\
\end{array}$ & 0.461 & $\begin{array}{c}2.70 \\
(1.95-3.20) \\
\end{array}$ & $\begin{array}{c}2.00 \\
(1.50-2.60) \\
\end{array}$ & 0.002 \\
\hline Triglycerides [mmol/l] & $\begin{array}{c}1.28 \\
(0.79-1.75) \\
\end{array}$ & $\begin{array}{c}0.93 \\
(0.79-1.81) \\
\end{array}$ & 0.841 & $\begin{array}{c}1.25 \\
(0.99-1.80) \\
\end{array}$ & $\begin{array}{c}1.47 \\
(1.04-1.92) \\
\end{array}$ & 0.134 \\
\hline Fast glucose [mmol/l] & $\begin{array}{c}5.06 \\
(4.62-5.32)\end{array}$ & $\begin{array}{c}4.68 \\
(4.30-5.29)\end{array}$ & 0.265 & $\begin{array}{c}4.83 \\
(4.35-5.22)\end{array}$ & $\begin{array}{c}4.78 \\
(4.35-5.42)\end{array}$ & 0.723 \\
\hline $\mathrm{HbA}_{1 \mathrm{c}}(\%)$ & $\begin{array}{c}5.60 \\
(5.28-5.93) \\
\end{array}$ & $\begin{array}{c}5.70 \\
(5.60-5.90) \\
\end{array}$ & 0.327 & $\begin{array}{c}5.65 \\
(5.38-5.93) \\
\end{array}$ & $\begin{array}{c}5.90 \\
(5.50-6.20) \\
\end{array}$ & 0.006 \\
\hline $\mathrm{BUN}[\mathrm{mmol} / \mathrm{l}]$ & $\begin{array}{c}4.14 \\
(3.70-5.64) \\
\end{array}$ & $\begin{array}{c}4.73 \\
(4.21-6.48) \\
\end{array}$ & 0.127 & $\begin{array}{c}5.16 \\
(4.68-5.92) \\
\end{array}$ & $\begin{array}{c}5.40 \\
(4.48-6.30) \\
\end{array}$ & 0.649 \\
\hline $\begin{array}{l}\text { Serum creatinine } \\
{[\mu \mathrm{mol} / \mathrm{l}]}\end{array}$ & $\begin{array}{c}62.8 \\
(53.3-72.6)\end{array}$ & $\begin{array}{c}60.5 \\
(54.2-78.6)\end{array}$ & 0.841 & $\begin{array}{c}65.6 \\
(57.8-74.4)\end{array}$ & $\begin{array}{c}68.0 \\
(58.5-79.1)\end{array}$ & 0.326 \\
\hline Uric acid $[\mu \mathrm{mol} / \mathrm{l}]$ & $\begin{array}{c}350.0 \\
(292.5-396.3) \\
\end{array}$ & $\begin{array}{c}295.0 \\
(247.3-401.8) \\
\end{array}$ & 0.231 & $\begin{array}{c}331.5 \\
(271.0-373.0) \\
\end{array}$ & $\begin{array}{c}347.0 \\
(277.3-417.5) \\
\end{array}$ & 0.230 \\
\hline $\mathrm{Na}^{+}[\mathrm{mmol} / \mathrm{l}]$ & $\begin{array}{c}141.5 \\
(139.9-142.7)\end{array}$ & $\begin{array}{c}141.1 \\
(140.2-142.9)\end{array}$ & 0.841 & $\begin{array}{c}142.0 \\
(140.1-142.6)\end{array}$ & $\begin{array}{c}141.1 \\
(139.4-142.5)\end{array}$ & 0.077 \\
\hline $\mathrm{K}^{+}[\mathrm{mmol} / \mathrm{l}]$ & $\begin{array}{c}4.00 \\
(3.73-4.25)\end{array}$ & $\begin{array}{c}3.90 \\
(3.80-4.18)\end{array}$ & 0.968 & $\begin{array}{c}3.80 \\
(3.70-4.10)\end{array}$ & $\begin{array}{c}4.00 \\
(3.80-4.30)\end{array}$ & 0.012 \\
\hline Serum albumin $[\mathrm{g} / \mathrm{l}]$ & $\begin{array}{c}39.5 \\
(38.1-43.2) \\
\end{array}$ & $\begin{array}{c}39.3 \\
(37.7-42.0) \\
\end{array}$ & 0.841 & $\begin{array}{c}39.4 \\
(36.9-41.4) \\
\end{array}$ & $\begin{array}{c}40.1 \\
(38.3-42.3) \\
\end{array}$ & 0.076 \\
\hline $\begin{array}{l}\text { Free triiodothyronine } \\
{[\mathrm{pg} / \mathrm{ml}]}\end{array}$ & $\begin{array}{c}2.79 \\
(2.64-3.01) \\
\end{array}$ & $\begin{array}{c}2.80 \\
(2.56-3.03) \\
\end{array}$ & 0.923 & $\begin{array}{c}2.95 \\
(2.70-3.12) \\
\end{array}$ & $\begin{array}{c}2.93 \\
(2.78-3.13) \\
\end{array}$ & 0.529 \\
\hline $\begin{array}{l}\text { Free tetraiodothyronine } \\
{[\mathrm{ng} / \mathrm{dl}]}\end{array}$ & $\begin{array}{c}1.06 \\
(0.98-1.16) \\
\end{array}$ & $\begin{array}{c}1.11 \\
(1.02-1.20) \\
\end{array}$ & 0.411 & $\begin{array}{c}1.12 \\
(1.02-1.25) \\
\end{array}$ & $\begin{array}{c}1.11 \\
(1.04-1.22) \\
\end{array}$ & 0.779 \\
\hline $\mathrm{STSH}[\mu \mathrm{IU} / \mathrm{ml}]$ & $\begin{array}{c}1.85 \\
(1.12-2.41) \\
\end{array}$ & $\begin{array}{c}2.48 \\
(1.76-4.55)\end{array}$ & 0.057 & $\begin{array}{c}2.05 \\
(1.25-3.25) \\
\end{array}$ & $\begin{array}{c}1.83 \\
(1.28-3.15)\end{array}$ & 0.875 \\
\hline Fibrinogen $[\mathrm{mg} / \mathrm{dl}]$ & $\begin{array}{c}254.0 \\
(237.5-293.5) \\
\end{array}$ & $\begin{array}{c}278.3 \\
(252.6-309.2) \\
\end{array}$ & 0.112 & $\begin{array}{c}240.3 \\
(206.4-299.4) \\
\end{array}$ & $\begin{array}{c}249.7 \\
(221.8-294.0) \\
\end{array}$ & 0.251 \\
\hline NT-BNP [pg/ml] & $\begin{array}{c}54.4 \\
(16.0-109.9)\end{array}$ & $\begin{array}{c}43.6 \\
(22.6-117.9)\end{array}$ & 0.872 & $\begin{array}{c}45.1 \\
(26.7-89.3)\end{array}$ & $\begin{array}{c}60.8 \\
(33.4-115.2)\end{array}$ & 0.201 \\
\hline
\end{tabular}


Table III. Cont.

\begin{tabular}{|lcccccc|}
\hline Parameter & \multicolumn{3}{c}{ First validation } & \multicolumn{3}{c|}{ Second validation } \\
\cline { 2 - 7 } & $\begin{array}{c}\text { Control } \\
(n=20)\end{array}$ & $\begin{array}{c}\text { CCS } \\
(n=20)\end{array}$ & $P$-value & $\begin{array}{c}\text { Control } \\
(n=48)\end{array}$ & $\begin{array}{c}\text { CCS } \\
(n=100)\end{array}$ & $P$-value \\
\hline Aspirin, $n(\%)$ & $6(30.0)$ & $19(95.0)$ & $<0.001$ & $24(50.0)$ & $94(94.0)$ & $<0.001$ \\
\hline Clopidogrel, $n(\%)$ & $3(15.0)$ & $15(75.0)$ & $<0.001$ & $4(8.3)$ & $69(69.0)$ & $<0.001$ \\
\hline Ticagrelor, $n(\%)$ & $0(0.0)$ & $0(0.0)$ & 1.000 & $0(0.0)$ & $10(10.0)$ & 0.055 \\
\hline ACEl/ARB, $n(\%)$ & $4(20.0)$ & $5(25.0)$ & 0.705 & $10(20.8)$ & $36(36.0)$ & 0.062 \\
\hline Statin, $n(\%)$ & $12(60.0)$ & $19(95.0)$ & 0.023 & $33(68.8)$ & $95(95.0)$ & $<0.001$ \\
\hline$\beta$-blocker & $4(20.0)$ & $6(30.0)$ & 0.465 & $14(29.2)$ & $62(62.0)$ & $<0.001$ \\
\hline CCB, $n(\%)$ & $6(30.0)$ & $7(35.0)$ & 0.736 & $11(22.9)$ & $38(38.0)$ & 0.068 \\
\hline
\end{tabular}

CCS - chronic coronary syndrome, ESR - erythrocyte sedimentation rate, AST - aspartate aminotransferase, ALT-alanine aminotransferase, $H D L-C$ - high-density lipoprotein cholesterol, LDL-C - low-density lipoprotein cholesterol, $H b A_{1 c}-$ glycosylated hemoglobin, BUN - blood urea nitrogen, sTSH - thyroid stimulating hormone, $C K M B$ - creatine kinase $M B, A C E I / A R B$ - angiotensin-converting enzyme inhibitors/ angiotensin receptor blockers, $C C B$ - calcium antagonist, $L V E D D$ - left ventricular end diastolic diameter, LVEF - left ventricular ejection fraction, NT-BNP-N-terminal proB-type natriuretic peptide.

A

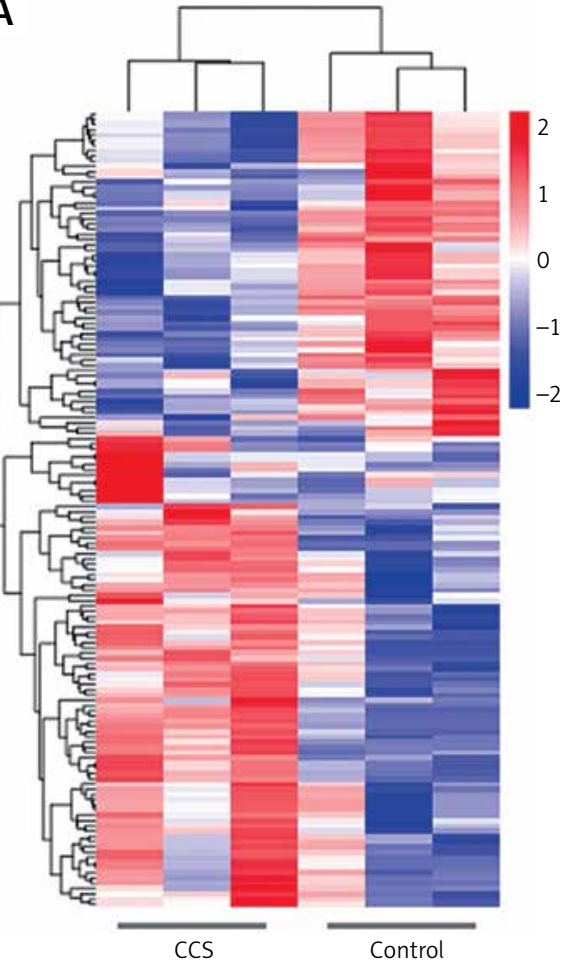

B

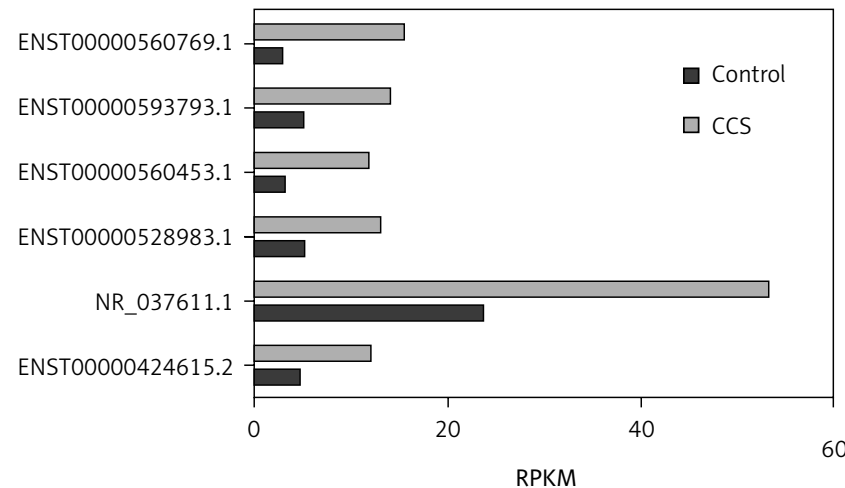

Figure 1. Circulating exosomal IncRNA sequencing in chronic coronary syndrome (CCS). A - Hierarchical clustering of substantially differentially expressed ( $p<0.05$; fold change $>2$ ) IncRNAs in control and CCS. B - Six upregulated IncRNAs in CCS were selected for the first validation

ENST-00000593793.1 $(p=0.442$, fold change $=1.29)$ were not (Figure 2). Therefore, the circulating exosomal IncRNAs ENST00000424615.2 and ENST000005-60769.1 were selected for the second validation.

\section{Second validation of circulating exosomal IncRNA}

The second validation was performed in $100 \mathrm{CCS}$ patients and 48 controls, whose clinical characteristics are displayed in Table III.
In the second validation, circulating exosomal IncRNA ENST00000424615.2 $(p=0.063$, fold change $=2.39)$ and IncRNA ENST00000560769.1 $(p=0.003$, fold change $=3.07)$ were both significantly upregulated in CCS patients compared with control subjects (Figure 3).

ROC curve analysis showed, for circulating exosomal IncRNA ENST00000424615.2, AUC $=0.654$ \pm 0.047 (95\% Cl: 0.562-0.746, $p=0.002)$; for IncRNA ENST00000560769.1, AUC = $0.722 \pm 0.048$ (95\% Cl: 0.627-0.817, $p<0.001)$ (Figure 3). 
A

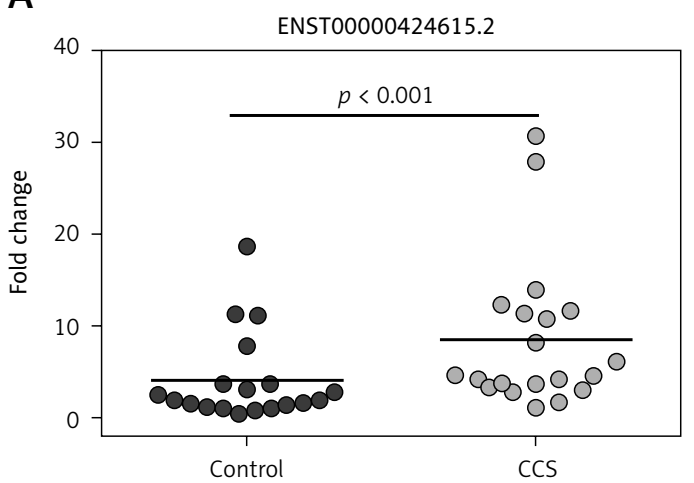

C

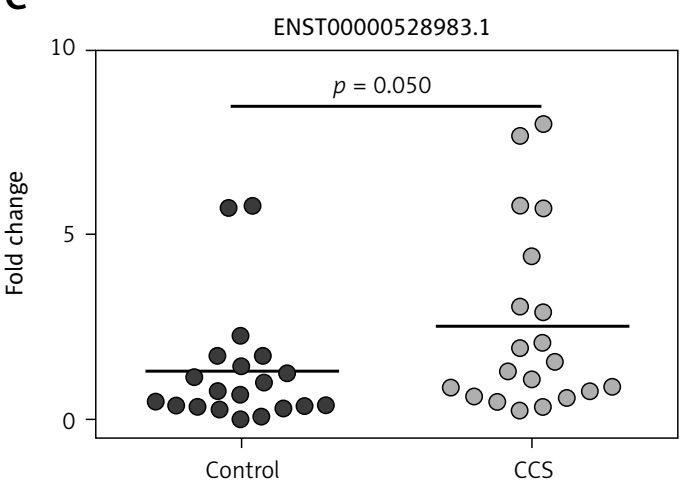

E

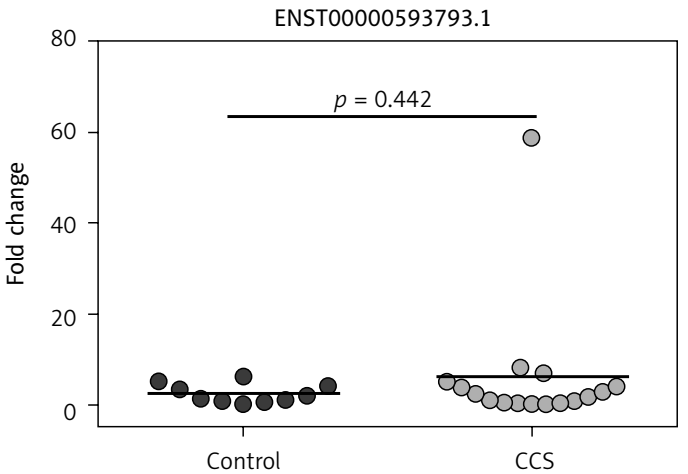

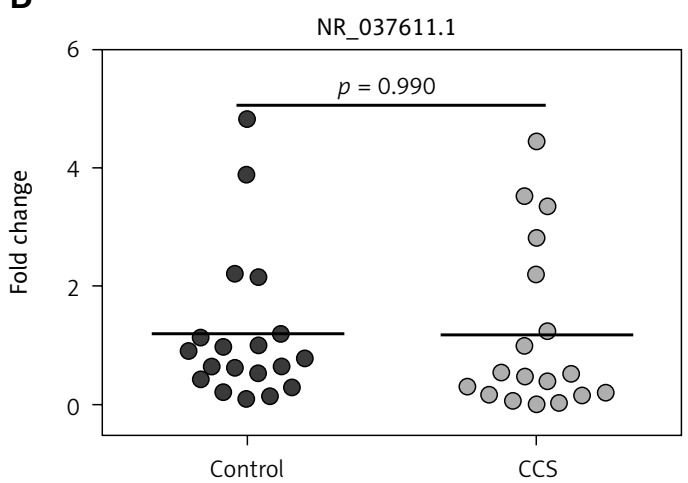

D

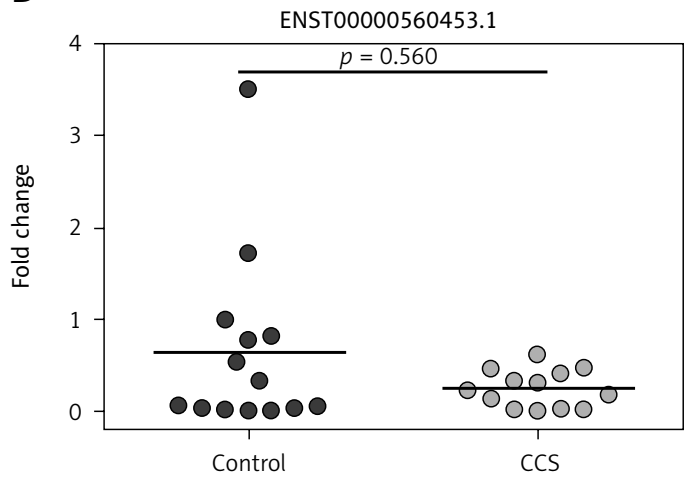

F

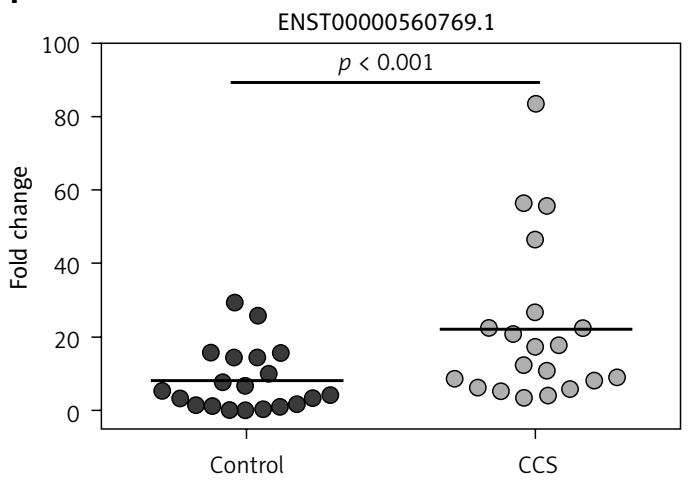

Figure 2. qRT-PCR analysis of expression of the six circulating exosomal IncRNAs (ENST00000424615.2, NR_037611.1, ENST00000528983.1, ENST00000560453.1, ENST00000593793.1, and ENST00000560769.1) that were selected in the first validation

Association between circulating exosomal IncRNA and clinical parameters

In the second validation, circulating exosomal IncRNAs ENST00000424615.2 and ENST00000560769.1 showed significant relationships with certain parameters in CCS patients. More specifically, exosomal IncRNA ENST00000424615.2 was positively associated with diastolic blood pressure $(r=0.191, p=0.020)$, and negatively associated with alanine aminotransferase (ALT) $(r=-0.224, p=0.028)$ (Table IV).
Exosomal IncRNA ENST00000560769.1 was significantly lower in the current drinker group ( $p=0.042$ ), but higher in the CCS patients with more diseased vessels $(p=0.028)$ (Table V).

\section{Discussion}

In the present study, we obtained interesting findings indicating that circulating exosomal IncRNAs ENST00000424615.2 and ENST00000560769.1 might act as potential biomarkers for CCS. Furthermore, exosomal IncRNA 
Table IV. Associations of circulating exosomal IncRNA ENST00000424615.2 and ENST00000560769.1 with clinical parameters of chronic coronary syndrome patients in the second validation

\begin{tabular}{|c|c|c|c|c|}
\hline \multirow[t]{2}{*}{ Parameter } & \multicolumn{2}{|c|}{ ENST00000424615.2 } & \multicolumn{2}{|c|}{ ENST00000560769.1 } \\
\hline & $r$ & $P$-value & $r$ & $P$-value \\
\hline Age & -0.077 & 0.448 & -0.024 & 0.813 \\
\hline Hospital time & 0.143 & 0.156 & -0.154 & 0.125 \\
\hline LVEDD & 0.100 & 0.362 & -0.033 & 0.765 \\
\hline LVEF & -0.082 & 0.453 & -0.039 & 0.720 \\
\hline Heart rate & 0.002 & 0.978 & -0.048 & 0.563 \\
\hline Systolic blood pressure & 0.055 & 0.504 & 0.025 & 0.759 \\
\hline Diastolic blood pressure & 0.191 & 0.020 & -0.023 & 0.781 \\
\hline Body mass index & -0.043 & 0.670 & -0.034 & 0.740 \\
\hline C-reactive protein & -0.166 & 0.111 & -0.151 & 0.146 \\
\hline ESR & -0.010 & 0.926 & 0.098 & 0.348 \\
\hline Leukocyte & 0.007 & 0.943 & -0.134 & 0.186 \\
\hline Neutrophil & 0.013 & 0.899 & -0.107 & 0.291 \\
\hline Lymphocyte & 0.045 & 0.655 & -0.103 & 0.311 \\
\hline Hemoglobin & 0.027 & 0.792 & 0.000 & 0.996 \\
\hline Platelets & -0.019 & 0.848 & -0.161 & 0.111 \\
\hline AST & -0.098 & 0.339 & 0.004 & 0.970 \\
\hline ALT & -0.224 & 0.028 & -0.085 & 0.409 \\
\hline Total cholesterol & 0.086 & 0.407 & -0.004 & 0.973 \\
\hline HDL-C & -0.080 & 0.443 & -0.004 & 0.968 \\
\hline LDL-C & 0.114 & 0.271 & -0.052 & 0.618 \\
\hline Triglycerides & 0.005 & 0.097 & 0.048 & 0.642 \\
\hline Fast glucose & -0.171 & 0.206 & 0.108 & 0.205 \\
\hline $\mathrm{HbA}_{1 \mathrm{c}}$ & -0.113 & 0.274 & 0.142 & 0.169 \\
\hline BUN & -0.073 & 0.479 & -0.093 & 0.367 \\
\hline Serum creatinine & 0.152 & 0.139 & 0.059 & 0.570 \\
\hline Uric acid & 0.152 & 0.139 & -0.061 & 0.556 \\
\hline $\mathrm{Na}^{+}$ & 0.197 & 0.054 & -0.019 & 0.857 \\
\hline $\mathrm{K}^{+}$ & 0.164 & 0.110 & 0.158 & 0.124 \\
\hline Serum albumin & -0.003 & 0.977 & 0.059 & 0.570 \\
\hline Free triiodothyronine & -0.005 & 0.965 & -0.157 & 0.133 \\
\hline Free tetraiodothyronine & -0.139 & 0.183 & -0.170 & 0.102 \\
\hline sTSH & -0.124 & 0.238 & 0.055 & 0.603 \\
\hline Fibrinogen & -0.034 & 0.738 & -0.089 & 0.381 \\
\hline NT-BNP & 0.102 & 0.423 & 0.090 & 0.481 \\
\hline
\end{tabular}

LVEDD - left ventricular end diastolic diameter, LVEF - left ventricular ejection fraction, ESR - erythrocyte sedimentation rate, AST aspartate aminotransferase, $A L T$ - alanine aminotransferase, HDL-C - high-density lipoprotein cholesterol, LDL-C - low-density lipoprotein cholesterol, $\mathrm{HbA}_{1 c}$ - glycosylated hemoglobin, BUN-blood urea nitrogen, sTSH - thyroid stimulating hormone, NT-BNP - N-terminal proBtype natriuretic peptide. 
Table V. Comparison of expression of circulating exosomal IncRNA ENST00000424615.2 and ENST00000560769.1 between different groups of clinical parameters in chronic coronary syndrome patients in the second validation

\begin{tabular}{|c|c|c|c|c|}
\hline \multirow[t]{2}{*}{ Parameter } & \multicolumn{2}{|c|}{ ENST00000424615.2 } & \multicolumn{2}{|c|}{ ENST00000560769.1 } \\
\hline & Median $\left(25^{\text {th }}-75^{\text {th }}\right)$ & $P$-value & Median $\left(25^{\text {th }}-75^{\text {th }}\right)$ & $P$-value \\
\hline Gender: & & 0.239 & & 0.159 \\
\hline Male & $5.90(2.07-14.53)$ & & $19.84(10.30-36.15)$ & \\
\hline Female & $2.97(0.83-19.56)$ & & $13.36(5.94-28.05)$ & \\
\hline Hypertension: & & 0.468 & & 0.327 \\
\hline No & $4.92(2.30-14.60)$ & & $19.83(10.93-34.54)$ & \\
\hline Yes & $4.89(1.04-15.89)$ & & $16.00(8.34-37.53)$ & \\
\hline Diabetes: & & 0.261 & & 0.381 \\
\hline No & $5.90(1.79-16.11)$ & & $16.80(9.04-34.66)$ & \\
\hline Yes & $2.97(1.04-5.39)$ & & $23.75(12.64-45.57)$ & \\
\hline Current smoker: & & 0.063 & & 0.652 \\
\hline No & $3.58(1.04-13.18)$ & & $15.35(8.69-49.87)$ & \\
\hline Yes & $6.96(2.88-17.15)$ & & $18.25(10.23-34.66)$ & \\
\hline Current drinker: & & 0.315 & & 0.042 \\
\hline No & $5.96(1.88-16.00)$ & & $20.69(10.54-42.84)$ & \\
\hline Yes & $3.85(0.96-14.11)$ & & $12.68(7.01-25.07)$ & \\
\hline Diseased vessel: & & 0.637 & & 0.028 \\
\hline$<3$ & $3.81(1.46-17.66)$ & & $12.91(7.02-24.38)$ & \\
\hline 3 or combined diseased LM & $5.90(1.65-14.53)$ & & $20.53(11.01-45.19)$ & \\
\hline
\end{tabular}

ENST00000560769.1 might be associated with CCS severity, as it was significantly higher in the CCS patients with more diseased vessels.

Increasing evidence has revealed that noncoding RNAs participate in various physiological and pathological processes, playing important roles in human diseases [19-21]. LncRNAs are noncoding RNAs longer than 200 nucleotides, which regulate gene expression at the transcriptional, post-transcriptional, and translational levels. Previous studies have proved that IncRNAs were involved in regulating pathophysiological conditions, such as cancer [22-24], autoimmune diseases [25], and others [26]. In CAD, IncRNAs were found to be differently expressed in atherosclerotic coronary artery plaques [27], and were proved to serve as biomarkers to diagnose CAD [11-14]. Several studies also reported that IncRNAs might be used for the prediction of ACS outcomes [28].

The IncRNA packaged in the exosomes are highly stable due to their lipid bilayers that protect them from enzymatic degradation by RNA enzymes in the bodily fluids. Thus, due to that high stability, exosomal IncRNAs are more suitable to be biomarkers. Previous studies showed that exosomal IncRNAs were potential biomarkers for various diseases [29-31]. In CAD, exosomal IncRNAs were reported to be involved in atherosclerosis progression [8-10], and they might serve as a valuable therapeutic tool for neovascularization [32].

Here, through sequencing profiles and double qRT-PCR validations, we established that circulating exosomal IncRNAs ENST00000424615.2 and ENST00000560769.1 were significantly upregulated in CCS patients. ENST00000424615.2, also named as RP11-38L15.2-001 (Ensembl Human GRCh37. p13), is a lincRNA, and a product of the gene ENSG00000229227.4, located in chromosome 10: 46,937,463-46,947,262 reverse strand. (Ensembl Human GRCh37. p13). ENST00000560769.1, also named as CTD-2033D15.1-001 (Ensembl Human GRCh37. p13), is an antisense IncRNA, and a product of the gene ENSG00000259279.1, located in chromosome 15: 39,885,781-39,886,432 reverse strand (Ensembl Human GRCh37. p13). These two IncRNAs are novel transcripts, on which no relevant research has been performed. In the present study, we found that circulating exosomal IncRNAs ENST00000424615.2andENST00000560769.1 were 
A

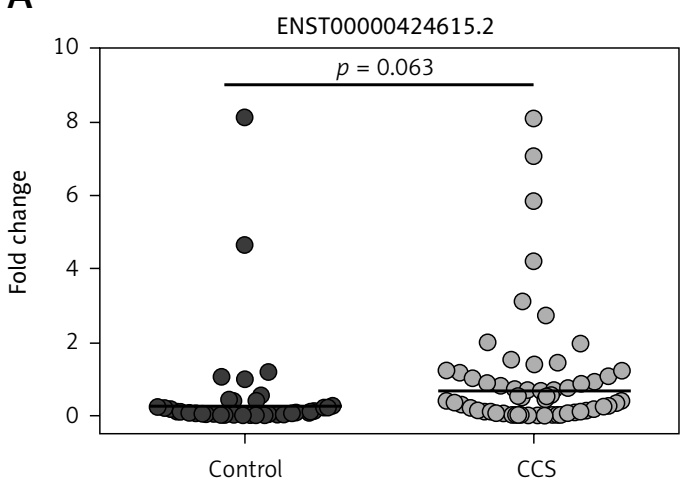

C

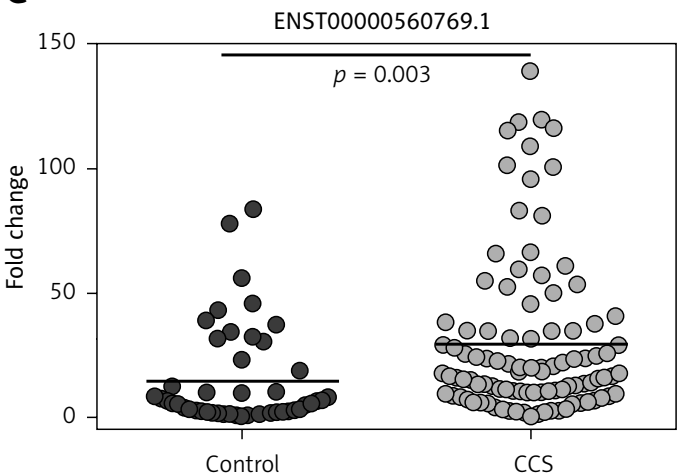

B

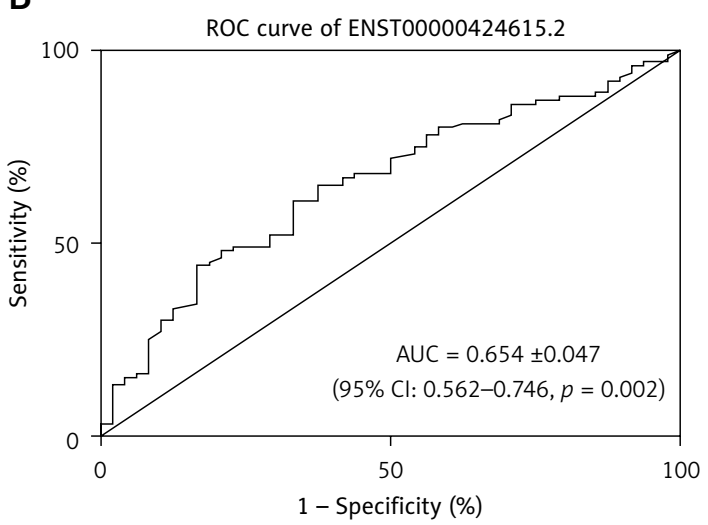

D

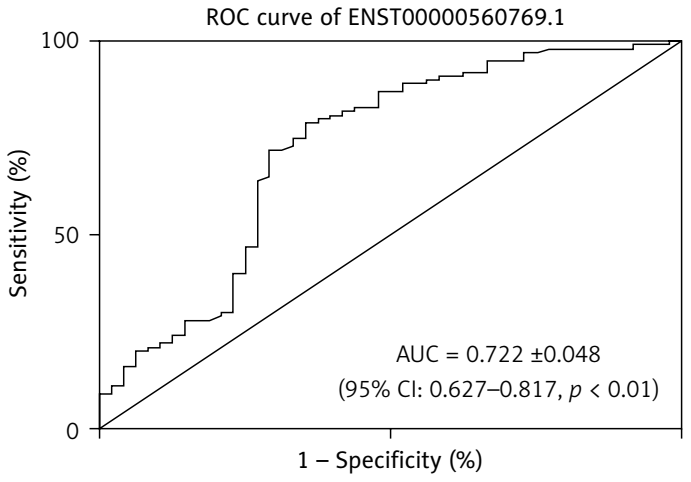

Figure 3. Circulating exosomal IncRNAs ENST00000424615.2 and ENST00000560769.1, selected in the second validation. A - qRT-PCR analysis of expression of ENST00000424615.2 and ENST00000560769.1. B - Receiver operating characteristic (ROC) curves analyses of ENST00000424615.2 and ENST00000560769.1 for chronic coronary syndrome

elevated in patients with CCS, and might serve as potential biomarkers for CCS. Moreover, exosomal IncRNA ENST00000560769.1 was significantly higher in the CCS patients with more diseased vessels, and might be associated with a poor prognosis.

Our results suggested that circulating exosomal IncRNAs may serve as potential biomarkers for CCS and even be associated with the severity of CCS. However, the present study also has some limitations: this study was a clinical observation, the mechanism by which circulating exosomal InCRNAs regulate CCS was not elucidated, and further research is needed to clarify these regulatory mechanisms; this study had a small sample size and lacked follow-up after discharge; thus the relationship between exosomal IncRNAs and prognosis was not evaluated, and a further large sample size study with long-term follow-up should be conducted.

In conclusion, our data showed that circulating exosomal IncRNAs ENST00000424615.2 and ENST00000560769.1 were elevated in patients with CCS, and might serve as potential biomarkers for CCS. Moreover, exosomal IncRNA ENST00000560769.1 was significantly higher in the CCS patients with more diseased vessels, and might be associated with a poor prognosis.

\section{Acknowledgments}

Meili Zheng, Ruijuan Han and Wen Yuan contributed equally to this work.

This research was supported by the National Natural Science Foundation of China (81800304, $81500038,81770253)$, the National Major Research Plan Training Program of China (91849111), and the Open Foundation from Beijing Key Laboratory of Hypertension Research (2019GXY-KFKT-03, 2019GXY-KFKT-02).

\section{Conflict of interest}

The authors declare no conflict of interest.

\section{References}

1. Knuuti J, Wijns W, Saraste A, et al. 2019 ESC guidelines for the diagnosis and management of chronic coronary syndromes. Eur Heart J 2020; 41: 407-77.

2. Gao XF, Wang ZM, Wang F, Gu Y, Zhang JJ, Chen SL. Exosomes in coronary artery disease. Int I Biol Sci 2019; 15: 2461-70.

3. Coumans FAW, Brisson AR, Buzas El, et al. Methodological guidelines to study extracellular vesicles. Circ Res 2017; 120: 1632-48.

4. Sluijter JPG, Davidson SM, Boulanger CM, et al. Extracellular vesicles in diagnostics and therapy of the ischae- 
mic heart: position paper from the Working Group on Cellular Biology of the heart of the European Society of Cardiology. Cardiovasc Res 2018; 114: 19-34.

5. Ghafarian F, Pashirzad M, Khazaei M, et al. The clinical impact of exosomes in cardiovascular disorders: from basic science to clinical application. J Cell Physiol 2019; 234: 12226-36

6. Bellin G, Gardin C, Ferroni L, et al. Exosome in cardiovascular diseases: a complex world full of hope. Cells 2019; 8: 166.

7. Su J, Li J, Yu Q, et al. Exosomal miRNAs as potential biomarkers for acute myocardial infarction. IUBMB Life 2020; 72: 384-400.

8. Huang C, Han J, Wu Y, et al. Exosomal MALAT1 derived from oxidized low-density lipoprotein-treated endothelial cells promotes M2 macrophage polarization. Mol Med Rep 2018; 18: 509-15.

9. Chen L, Yang W, Guo Y, et al. Exosomal IncRNA GAS5 regulates the apoptosis of macrophages and vascular endothelial cells in atherosclerosis. PLoS One 2017; 12 e0185406.

10. Wang Y, Liang J, Xu J, et al. Circulating exosomes and exosomal IncRNA HIF1A-AS1 in atherosclerosis. Int J Clin Exp Pathol 2017; 10: 8383-8.

11. Zhang Z, Gao W, Long QQ, et al. Increased plasma levels of IncRNA H19 and LIPCAR are associated with increased risk of coronary artery disease in a Chinese population. Sci Rep 2017; 7: 7491.

12. Yin $\mathrm{Q}$, Wu A, Liu M. Plasma long non-coding RNA (IncRNA) GAS5 is a new biomarker for coronary artery disease. Med Sci Monit 2017; 23: 6042-8.

13. Cai Y, Yang Y, Chen X, et al. Circulating 'IncRNA OTTHUMT00000387022' from monocytes as a novel biomarker for coronary artery disease. Cardiovasc Res 2016; 112: 714-24.

14. Li L, Wang L, Li H, et al. Characterization of IncRNA expression profile and identification of novel IncRNA biomarkers to diagnose coronary artery disease. Atherosclerosis 2018; 275: 359-67.

15. Li L, Li C, Wang S, et al. Exosomes derived from hypoxic oral squamous cell carcinoma cells deliver MIR-21 to normoxic cells to elicit a prometastatic phenotype. Cancer Res 2016; 76: 1770-80.

16. Zhang L, Li H, Yuan M, Li D, Sun C, Wang G. Serum exosomal microRNAs as potential circulating biomarkers for endometriosis. Dis Markers 2020; 2020: 2456340.

17. Zhang L, Zhang S, Yao J, et al. Microenvironmentinduced PTEN loss by exosomal microRNA primes brain metastasis outgrowth. Nature 2015; 527: 100-4.

18. Zheng $M$, Zhao L, Yang X. Expression profiles of long noncoding RNA and mRNA in epicardial adipose tissue in patients with heart failure. Biomed Res Int 2019; 2019: 3945475.

19. Yu P, Guo J, Li J, Chen W, Zhao T. Co-expression network analysis revealing the key IncRNAs in diabetic foot ulcers Arch Med Sci 2019; 15: 1123-32.

20. Zhou JZ, Hu MR, Diao HL, Wang QW, Huang Q, Ge BJ. Comprehensive analysis of differentially expressed circRNAs revealed a ceRNA network in pancreatic ductaladenocarcinoma. Arch Med Sci 2019; 15: 979-91.

21. El-Ahwany EGE, Mourad L, Zoheiry MMK, et al. MicroRNA-122a as a non-invasive biomarker for HCV genotype 4-related hepatocellular carcinoma in Egyptian patients. Arch Med Sci 2019; 15: 1454-61.

22. Shao S, Tian J, Zhang H, Wang S. LncRNA myocardial infarction-associated transcript promotes cell proliferation and inhibits cell apoptosis by targeting MIR-330-5p in epithelial ovarian cancer cells. Arch Med Sci 2018; 14 1263-70.

23. Zhang S, Song X. Long non-coding RNA SNHG1 promotes cell proliferation and invasion of hepatocellular carcinoma by acting as a molecular sponge to modulate MIR-195. Arch Med Sci 2020; 16: 386-94.

24. Liu H, Liu J, Zhao G. Long non-coding RNA HOTAIR regulates proliferation, migration and invasion of human cervical cancer cells by modulating expression of MAPK1. Arch Med Sci 2020; 16: 1158-65.

25. Zhang $\mathrm{Q}$, Liang $\mathrm{Y}$, Yuan $\mathrm{H}$, et al. Integrated analysis of IncRNA, miRNA and mRNA expression profiling in patients with systemic lupus erythematosus. Arch Med Sci 2019; 15: 872-9.

26. Yang $M$, Zhang L, Wang X, Zhou Y, Wu S. Down-regu lation of MIR-203a by IncRNA PVT1 in multiple myeloma promotes cell proliferation. Arch Med Sci 2018; 14 1333-9.

27. Arslan S, Berkan O, Lalem T, et al. Long non-coding RNAs in the atherosclerotic plaque. Atherosclerosis 2017; 266 176-81.

28. Vausort M, Wagner DR, Devaux Y. Long noncoding RNAs in patients with acute myocardial infarction. Circ Res 2014; 115: 668-77.

29. Zhao Y, Xu J. Synovial fluid-derived exosomal IncRNA PCGEM1 as biomarker for the different stages of osteoarthritis. Int Orthop 2018; 42: 2865-72.

30. Lee YR, Kim G, Tak WY, et al. Circulating exosomal non coding RNAs as prognostic biomarkers in human hepatocellular carcinoma. Int J Cancer 2019; 144: 1444-52.

31. Zhao R, Zhang Y, Zhang X, et al. Exosomal long noncoding RNA HOTTIP as potential novel diagnostic and prognostic biomarker test for gastric cancer. Mol Cancer 2018; 17: 68.

32. Shyu KG, Wang BW, Pan CM, Fang WJ, Lin CM. Hyperbaric oxygen boosts long noncoding RNA MALAT1 exosome secretion to suppress microRNA-92a expression in therapeutic angiogenesis. Int J Cardiol 2019; 274: 271-8. 